\title{
Effect of Classroom Illuminance on the Development and Progression of Myopia in School Children
}

\author{
Young-Woo Suh, Suk-Gyu Ha, Seung-Hyun Kim \\ Department of Ophthalmology, Korea University College of Medicine, Seoul, Korea
}

\begin{abstract}
Purpose: To evaluate the effect of classroom illuminance on the development and progression of myopia in elementary school children.

Methods: The daylight factor, a ratio of inside and outside illuminance, was obtained in 50 elementary schools. The firstgrade students in the school with the lowest daylight (LD) factor (LD school, 145 subjects; 0.51\%) and with the highest daylight (HD) factor (HD school, 147 subjects; 13.35\%) were selected. A survey was conducted to evaluate parental myopia, the amount of near-work and outdoor activities. The refractive error and axial length (AL) were measured at initial and after 6 months. The spherical equivalent, $\mathrm{AL}$, and the survey results were compared between the two schools. The mean $\mathrm{AL}$ of the emmetropic children was obtained, and all subjects were divided into two groups, more and less than mean AL. Changes in refractive errors and $\mathrm{AL}$ were also compared according to $\mathrm{AL}$.

Results: The amount of change in spherical equivalent and AL after 6 months were not different between the two schools. Initial prevalence of myopia was high in the HD school. However, it became similar between the two schools after 6 months. The mean AL of 155 emmetropic children was $22.7 \pm 0.63 \mathrm{~mm}$. In the 185 children with $\mathrm{AL} \geq 22.7 \mathrm{~mm}$, there was no difference in the AL change between the two schools. However, the change in AL in 107 children with $A L<22.7$ mm was significantly larger in the LD school $(0.19 \mathrm{~mm})$ than that in the $\mathrm{HD}$ school $(0.15 \mathrm{~mm}, p=0.049)$. Parental myopia, near-work and outdoor activities were not different between the two schools.
\end{abstract}

Conclusions: High classroom illuminance during the day reduced axial elongation in eyes of children with a shorter AL. Increase in classroom light level by permitting more sunlight can be a protective measure against the development of myopia.

Key Words: Child health, Myopia, Ocular vision, Public health

Uncorrected refractive error is the most common etiology for visual impairment worldwide [1]. Among refractive

Received: October 28, 2021 Final revision: December 15, 2021 Accepted: December 28, 2021

Corresponding Author: Seung-Hyun Kim, MD, PhD. Department of Ophthalmology, Korea University College of Medicine, 73 Inchon-ro, Seongbuk-gu, Seoul 02841, Korea. Tel: 82-2-920-5520, Fax: 82-2-9246820, E-mail: ansaneye@hanmail.net errors, myopia is highly prevalent and develops in $80 \%$ to $90 \%$ of school children, particularly in East and Southeast Asia [2]. Myopia is becoming a major public health problem, as it causes increased public health costs for glasses, contact lenses, and refractive surgery [3]. High myopia also increases the risk of retinal detachment, myopic macular degeneration, and glaucoma $[4,5]$. The incidence and prevalence of myopia is increasing, and Holden et al. [6] reported that $49.8 \%$ of the world's population will have myopia 
in 2050. Numerous studies on the mechanism of development and progression of myopia have been performed [7], and various attempts have been made to prevent or retard myopic progression, such as atropine eye drops [8] and orthokeratology [9]. Parental myopia and excessive nearwork are major causes of myopia [10-13]. Recently, outdoor activity was documented to show protective effect against myopia $[10,14,15]$. Wu et al. [16] reported that increasing outdoor activity by forcing school children to spend time outdoors during class recess reduces the development of myopia in nonmyopic school children, although it did not reduce the progression of myopia in myopic children. The mechanism of reduction in myopic progression by increasing outdoor activity is still unclear. It has been suggested that increased release of the retinal transmitter dopamine after sunlight exposure suppresses myopic development [17]. Other researchers have argued that miosis induced by bright sunlight increases depth of focus and subsequently decreases peripheral hyperopic retinal defocus, which has been reported to induce myopia $[18,19]$.

School children now spend more time indoors and are exposed to more indoor illumination. Animal experiments have revealed that elevated light levels (10,000-25,000 lux) in the laboratory slow the normal decrease in hyperopia $[17,20,21]$. Smith et al. [22] reported that high ambient light reduces form-deprivation myopia in rhesus monkeys and suggested that increasing indoor lighting level may be therapeutic protection against myopia. However, the effect of indoor illumination on myopia has not been studied well in humans. The present study was conducted to investigate the effect of classroom illumination on the development and progression of myopia in elementary school children.

\section{Materials and Methods}

This study protocol was reviewed and approved by the Institutional Review Board of Korea University Anam Hospital and adhered to the tenets of the Declaration of Helsinki. Among the first-grade elementary school children (6-7 years old), those whose parents agreed for them to participate in this study were enrolled. Informed consent was obtained from all children and their parents, and ophthalmologic examinations were performed.

The classroom and outdoor illuminance values were obtained in 50 elementary schools in South Korea (Seoul,
Gyunggido, Daejeon, and Chungchungdo) which permitted measurements of illuminance. The outdoor and classroom illuminance were measured at 10:30 to 11:00 a.m. and 2:30 to 3:00 p.m. considering that the sun reaches the meridian transit altitude at 12:35 p.m. The incidence angle of sunlight was the same between these two time periods. Classroom illuminance was measured at nine points on the desk plane (Fig. 1) of all classrooms, and the mean value was obtained. All indoor and outdoor illuminance values were measured by Korea Institute of Lighting Technology using an illuminometer (UA-002-64; Onset Hobo, Bourne, MA, USA). The daylight factor, which was defined as the ratio of the illuminance at a point on the working plane and outdoor illuminance, was calculated (mean value of indoor illuminance [lux] at nine points on the desk plane / outdoor illuminance $[\mathrm{lux}] \times 100 \%$ ). Indoor light level in the classroom is strongly affected by sunlight through windows because sunlight is a far more intense light source than artificial lighting. So, classroom illuminance varies according to the outdoor light level (time and weather). However, the daylight factor is relatively constant and re-

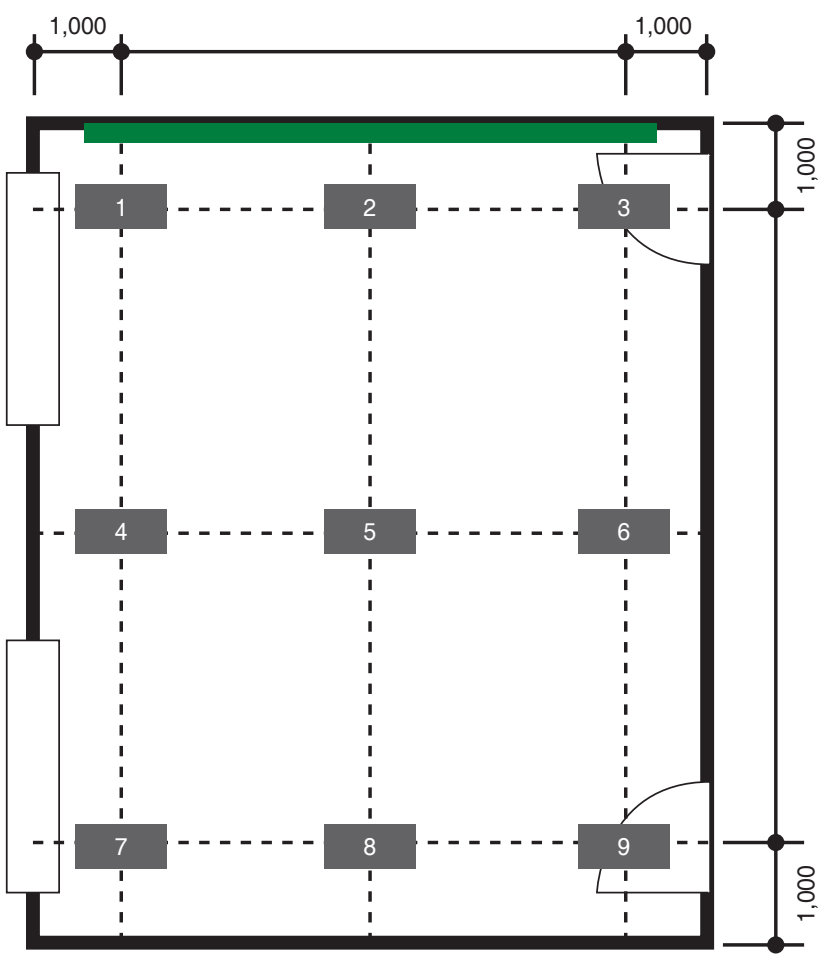

Fig. 1. The nine measurement points to determine classroom illuminance at the desk plane. The daylight factor was calculated as the following: classroom daylight factor $=$ mean value of indoor illuminance (lux) at nine points on the desk plane / outdoor illuminance (lux) $\times 100 \%$. 
flects indoor light level regardless of outdoor light intensity because it is the ratio of indoor illuminance through the window to outdoor illuminance. When outdoor illuminance increases, indoor illuminance through the window also increases, so the ratio remains relatively constant. Among the 50 elementary schools, the school with the lowest daylight (LD) factor (LD school; daylight factor, 0.51\%) and that with the highest daylight (HD) factor (HD school; daylight factor, 13.35\%) were selected. Both schools were located in metropolitan cities with populations more than a million. The north latitude of LD school is $37^{\circ}$ and that of HD school is $36^{\circ}$.

Presenting visual acuity was measured with a Snellen acuity chart, and the anterior segment was examined with a slit-lamp (Kowa Optimed, Torrance, CA, USA). Subjects with anterior segment abnormalities, such as corneal opacity or cataracts, were excluded. Refractive error was measured with an autokeratorefractometer (KR-800; Topcon, Tokyo, Japan), and the spherical equivalent value (SE) was obtained. Axial length (AL) was measured with IOL Master (Carl Zeiss Meditec, Jena, Germany). A survey was conducted to evaluate children's history for ophthalmologic care, parental myopia, and the amount of near-work and outdoor activity (Table 1).
Trivial diseases that did not affect refractive error or visual acuity, such as temporary conjunctivitis, hordeolum, and chalazion, were not included. The ophthalmologic examination was performed 1 to 2 months after school admission (April to May) and was repeated 6 months after the first examination (October to November). There was summer vacation between the two measurements. The values obtained from the right eye were used for analysis. Myopia was defined as SE $\leq-0.50$ diopters (D). The SE, prevalence of myopia, and AL were compared between the two schools at each time point and the amount of change was compared. The ophthalmologic history, parental myopia, and the amount or near-work and outdoor activities were compared between the two schools using the survey results. Initial AL of emmetropic children whose initial SE was -0.25 to $+1.00 \mathrm{D}(\mathrm{n}=155)$ was $22.7 \pm 0.63 \mathrm{~mm}$. We compared changes in ophthalmologic parameters in children with AL values $<22.7 \mathrm{~mm}$ and $\geq 22.7 \mathrm{~mm}$ separately to determine whether illuminance can affect children's refractive error differently according to AL [16,23].

The statistical analysis was performed using IBM SPSS ver. 21.0 (IBM Corp., Armonk, NY, USA). Student $t$-test and Mann-Whitney $U$-test were performed to compared $\mathrm{SE}, \mathrm{AL}$, the amount of change, and the amount of near-

Table 1. Questionnaire to evaluate children's history for ophthalmologic care, parental myopia, and the amount of near-work and outdoor activity

\begin{tabular}{l}
\hline 1. Has your child undergone eye surgery? \\
※ if any, \\
$\square$ Strabismus $\square$ Ptosis $\square$ Yes $\square$ No \\
2. Has your child been diagnosed with ocular diseases? \\
※ If any, \\
$\square$ Strabismus $\square$ Amblyopia $\square$ Ptosis $\square$ Epiblepharon $\square$ Others ( ) \\
3-1. Dose the child's father have myopia? \\
3-2. Does the child's mother have myopia? \\
※ How do you know you have myopia? \\
Myopia means near sightedness. If you have myopia, you can see near things well, but images blur when you are looking at far \\
things, assuming that you are <40 years of age. If you wear glasses, you can check with your glasses. If you place your glasses near \\
a newspaper and the letters look smaller than their original size, you are wearing glasses to correct for myopia. \\
4. How many times does your child read books for more than 30 minutes per week? \\
5. How many hours does your child read books per week? \\
6. How many hours does your child use a computer per week? \\
7. How many hours does your child use a smart phone per week? \\
8. How many times does your child do outdoor activity per week? \\
9. How many hours does your child do outdoor activity per week? \\
10. How many hours does your child expose to the sun light per week? \\
\hline
\end{tabular}


work and outdoor activity between the two schools as appropriate. Changes after 6 months were compared using the paired $t$-test and Wilcoxon signed-rank test. Ophthalmologic history and parental myopia were compared using the chi-square test. A $p$-value $<0.05$ was considered significant.

\section{Results}

At the initial examination, 162 children from the LD school and 153 children from the HD school participated. Among them, the ophthalmologic examination was successfully performed in 145 from the LD school and 147

Table 2. The presence of parental myopia, amount of near-work, and outdoor activity in all subjects

\begin{tabular}{|c|c|c|c|c|}
\hline Variable & $\begin{array}{l}\text { LD school } \\
(\mathrm{n}=140)\end{array}$ & $\begin{array}{l}\text { HD school } \\
(\mathrm{n}=144)\end{array}$ & Total & $p$-value \\
\hline Parental myopia & & & & 0.539 \\
\hline None & $42(30.0)$ & $52(36.1)$ & $94(33.0)$ & \\
\hline One parent & $44(31.4)$ & $40(27.8)$ & $84(29.5)$ & \\
\hline Both parents & $54(38.6)$ & $52(36.1)$ & $106(37.3)$ & \\
\hline \multicolumn{5}{|l|}{ Near-work activity } \\
\hline No. of times reading books for more than $30 \mathrm{~min}$ in a week & $4.36 \pm 2.50$ & $4.81 \pm 4.06$ & $4.58 \pm 3.38$ & 0.539 \\
\hline Hours of reading books in a week & $3.60 \pm 2.74$ & $4.36 \pm 4.86$ & $3.98 \pm 3.97$ & 0.700 \\
\hline Hours of using computers in a week & $1.50 \pm 2.05$ & $1.23 \pm 2.25$ & $1.36 \pm 2.16$ & 0.070 \\
\hline Hours of using smart phone in a week & $1.56 \pm 2.98$ & $1.70 \pm 3.17$ & $1.63 \pm 3.07$ & 0.462 \\
\hline \multicolumn{5}{|l|}{ Outdoor activity } \\
\hline No. of times of outdoor activity in a week & $3.30 \pm 4.27$ & $3.43 \pm 1.86$ & $3.37 \pm 3.28$ & 0.036 \\
\hline Hours of outdoor activity in a week & $4.05 \pm 3.25$ & $4.19 \pm 3.62$ & $4.12 \pm 3.43$ & 0.929 \\
\hline Hours of sun exposure in a week & $4.24 \pm 3.65$ & $4.64 \pm 6.27$ & $4.44 \pm 5.14$ & 0.771 \\
\hline
\end{tabular}

Values are presented as number (\%) or mean \pm standard deviation.

$\mathrm{LD}=$ lowest daylight; $\mathrm{HD}=$ highest daylight.

Table 3. Comparison of refractive errors, axial length, and prevalence of myopia between the two schools at initial examination and after 6 months

\begin{tabular}{|c|c|c|c|c|}
\hline Variable & LD school & HD school & Total & $p$-value \\
\hline \multicolumn{5}{|c|}{ Spherical equivalent (D) } \\
\hline Initial & $-0.09 \pm 0.86$ & $-0.41 \pm 0.92$ & $-0.25 \pm 0.90$ & 0.002 \\
\hline After 6 months & $-0.34 \pm 1.03$ & $-0.64 \pm 1.07$ & $-0.49 \pm 1.06$ & 0.014 \\
\hline Amount of change & $0.25 \pm 0.63$ & $0.23 \pm 0.82$ & $0.24 \pm 0.73$ & 0.843 \\
\hline$p$-value & $<0.001$ & 0.001 & $<0.001$ & - \\
\hline \multicolumn{5}{|l|}{ Axial length (mm) } \\
\hline Initial & $22.83 \pm 0.78$ & $22.96 \pm 0.83$ & $22.89 \pm 0.81$ & 0.148 \\
\hline After 6 months & $22.98 \pm 0.80$ & $23.15 \pm 0.89$ & $23.07 \pm 0.85$ & 0.094 \\
\hline Amount of change & $0.16 \pm 0.21$ & $0.18 \pm 0.17$ & $0.17 \pm 0.19$ & 0.189 \\
\hline$p$-value & $<0.001$ & 0.029 & $<0.001$ & - \\
\hline \multicolumn{5}{|c|}{ No. of myopic subjects ${ }^{*}$} \\
\hline Initial & $37(25.5)$ & $55(37.4)$ & $96(32.9)$ & 0.029 \\
\hline After 6 months & $55(37.9)$ & $66(44.9)$ & $121(41.4)$ & 0.227 \\
\hline
\end{tabular}

Values are presented as mean \pm standard deviation or number (\%).

$\mathrm{LD}=$ lowest daylight; $\mathrm{HD}=$ highest daylight; $\mathrm{D}=$ diopters .

${ }^{*}$ Spherical equivalent $\leq-0.50 \mathrm{D}$. 
from the HD school after 6 months. The data of these 292 children were used for analysis. There were 75 boys (51.7\%) in the LD school, and 78 (53.1\%) in the HD school, and no difference in the sex distribution was detected between the two schools $(p=0.981)$.

Nine children (two with amblyopia, four with strabismus, two with nasolacrimal duct obstruction, and one with a dermoid) at the LD school had an ophthalmologic history. Only one had undergone surgery (dermoid excision). Two children at the HD school had amblyopia, one had strabismus, and three had epiblepharon (two were surgically corrected) $(p=0.411)$. All children with either strabismus or amblyopia showed presenting visual acuity $\geq 0.7$. Parents of 284 children answered the survey about parental myopia. The numbers of children with both parents without myopia, one parent with myopia, and both parents with myopia were 43,44 , and 54 , respectively in the LD school, and 52, 40, and 52 in the HD school ( $p=0.539)$ (Table 2). The amount of near-work, including reading books and using a computer or smart phone was not different between the two schools (Table 2). More time was spent outdoors per week by children at the HD school ( $p=0.036)$, but the difference was only 0.1 times per week, which was too small to induce any change.

Table 3 shows the changes in SE, AL, and prevalence of myopia. At the initial examination, $\mathrm{SE}$ was more myopic at the HD school ( $p=0.002)$, but no difference in AL values were observed between the two schools. The amount of change in SE and AL after 6 months was not different between the schools. The initial prevalence of myopia was higher in the HD school than that in the LD school. However, the prevalence increased in the LD school, and it be-

Table 4. Comparison of refractive errors and axial length between the two schools at the initial examination and after 6 months according to the axial length

\begin{tabular}{|c|c|c|c|c|}
\hline Variable & LD school & HD school & Total & $p$-value \\
\hline \multicolumn{5}{|l|}{ Axial length $\geq 22.7 \mathrm{~mm}$} \\
\hline \multicolumn{5}{|c|}{ Spherical equivalent (D) } \\
\hline Initial & $-0.34 \pm 0.78$ & $-0.67 \pm 0.98$ & $-0.52 \pm 0.91$ & 0.031 \\
\hline After 6 months & $-0.47 \pm 1.04$ & $-0.82 \pm 1.12$ & $-0.66 \pm 1.09$ & 0.036 \\
\hline Amount of change & $0.13 \pm 0.63$ & $0.15 \pm 0.78$ & $0.14 \pm 0.71$ & 0.408 \\
\hline$p$-value & 0.033 & 0.016 & 0.001 & - \\
\hline \multicolumn{5}{|l|}{ Axial length (mm) } \\
\hline Initial & $23.38 \pm 0.55$ & $23.45 \pm 0.61$ & $23.42 \pm 0.58$ & 0.388 \\
\hline After 6 months & $23.51 \pm 0.63$ & $23.66 \pm 0.67$ & $23.60 \pm 0.65$ & 0.179 \\
\hline Amount of change & $0.14 \pm 0.24$ & $0.21 \pm 0.16$ & $0.18 \pm 0.21$ & 0.073 \\
\hline$p$-value & $<0.001$ & $<0.001$ & $<0.001$ & - \\
\hline \multicolumn{5}{|l|}{ Axial length $<22.7 \mathrm{~mm}$} \\
\hline \multicolumn{5}{|c|}{ Spherical equivalent (D) } \\
\hline Initial & $0.21 \pm 0.84$ & $0.01 \pm 0.63$ & $0.12 \pm 0.76$ & 0.173 \\
\hline After 6 months & $-0.18 \pm 1.00$ & $-0.35 \pm 0.94$ & $-0.26 \pm 0.98$ & 0.651 \\
\hline Amount of change & $0.39 \pm 0.59$ & $0.36 \pm 0.88$ & $0.28 \pm 0.73$ & 0.351 \\
\hline$p$-value & $<0.001$ & $<0.001$ & $<0.001$ & - \\
\hline \multicolumn{5}{|l|}{ Axial length (mm) } \\
\hline Initial & $22.18 \pm 0.45$ & $22.17 \pm 0.45$ & $22.18 \pm 0.45$ & 0.942 \\
\hline After 6 months & $22.37 \pm 0.46$ & $22.32 \pm 0.49$ & $22.35 \pm 0.47$ & 0.517 \\
\hline Amount of change & $0.19 \pm 0.16$ & $0.15 \pm 0.18$ & $0.17 \pm 0.17$ & 0.049 \\
\hline$p$-value & $<0.001$ & 0.002 & $<0.001$ & - \\
\hline
\end{tabular}

Values are presented as mean \pm standard deviation.

$\mathrm{LD}=$ lowest daylight; $\mathrm{HD}=$ highest daylight; $\mathrm{D}=$ diopters . 
came similar after 6 months $(p=0.227)$, suggesting that development of new myopia was higher in the LD school among previously nonmyopic children with a shorter AL (Table 3).

Thus, using mean AL of emmetropic children (155 children, SE $>-0.25 \mathrm{D}$ and $<+1.00 \mathrm{D}$ ), which was $22.7 \pm 0.63$ $\mathrm{mm}$, we analyzed children with $\mathrm{AL}<22.7 \mathrm{~mm}$ and $\geq 22.7$ $\mathrm{mm}$ separately. No difference in the change in AL was observed between the two schools in children with $\mathrm{AL} \geq 22.7$ $\mathrm{mm}$ (Table 4). However, the amount of change in AL after 6 months was significantly larger in the LD school (0.19 $\mathrm{mm})$ than that in the HD school $(0.15 \mathrm{~mm})$ in children with $\mathrm{AL}<22.7 \mathrm{~mm}(p=0.049)$ (Table 4). The number of children whose AL increased more than the mean $(0.17 \mathrm{~mm})$ was significantly larger in the LD school (31 children, $46.3 \%)$ than in the HD school (15 children, 26.8\%) among children with initial $\mathrm{AL}<22.7 \mathrm{~mm}(p=0.026)$. No difference in children with initial $\mathrm{AL} \geq 22.7 \mathrm{~mm}$ was detected between the two schools $(p=0.273)$.

\section{Discussion}

This study showed changes in SE and AL in two schools with different indoor illuminance and other known factors related with myopia. Children with myopic parents have a greater chance of developing myopia [13]. Many studies showed a relationship between myopia and near-work. Saw et al. [12] reported children who read more than two books per week have a higher degree of myopia than those who do not. Lee et al. [11] noted that increased time spent reading induces more myopia. Outdoor activity is thought to play a protective role against myopia [10,11,15,17,24]. Rose et al. [15] reported that the amount of time spent outdoors is more important than the activity itself. $\mathrm{Wu}$ et al. [16] documented that outdoor activity during class recess decreased the development of myopia in a prospective study. We investigated the presence of parental myopia, amount of near-work and outdoor activity in addition to indoor illuminance to adjust the effect on myopia. According to our study, children in classrooms with low illuminance showed a greater increase in AL when their initial AL was $<22.7$ $\mathrm{mm}$ (mean for emmetropic children). Classroom illuminance did not affect myopia in children with a longer initial AL. Factors other than classroom illuminance, such as the presence of parental myopia, the amount of near-work and outdoor activity, were not different between the two schools.

Wu et al. [16] noted that the effect of outdoor activity differs according to the initial refractive status of children. Outdoor activity during class recess decreases the shift in refractive error toward myopia in nonmyopic children, but does not retard progression of myopia in children who already are myopic. In our study, we could not perform cycloplegic refraction because many parents did not give consent. Although manifest refraction has been used in studies on myopia, it can be inaccurate in some children with various degrees of accommodation. We decided to use $\mathrm{AL}$ as a reference and divided the children into longer and shorter AL groups. In our study population, mean $\mathrm{AL}$ of emmetropic children was $22.7 \mathrm{~mm}$. Mutti et al. [25] reported that $\mathrm{AL}$ of emmetropic children (SE, -0.25 to 1.00 D) in their study population (age, 6 to 14 years) was also $22.7 \mathrm{~mm}$. We used AL of $22.7 \mathrm{~mm}$ as a reference point and divided the children according to this value. Our results correspond well with Wu et al. [16] in which light reduced axial elongation of the eye in nonmyopic school children.

Hua et al. [23] also investigated the effect of classroom illuminance on myopia. They selected two schools in which the classrooms did not fulfill the required classroom illuminance of 300 lux. Artificial lighting was used to increase the illuminance to 558 lux, and the effect of lighting was compared between brighter and darker classrooms. They reported that nonmyopic children from the brighter classroom showed less refractive shift toward myopia. Axial elongation was $0.13 \mathrm{~mm} / \mathrm{yr}$ in the high illuminance classroom and $0.18 \mathrm{~mm} / \mathrm{yr}$ in low illuminance classroom among nonmyopic children, which were $0.15 \mathrm{~mm} / 0.5 \mathrm{yr}$ and $0.19 \mathrm{~mm} / 0.5 \mathrm{yr}$ in our study. However, the study setting was different. Hua et al. [23] increased classroom illuminance from 74 to 558 lux using a lighting system, and illuminance was measured at 8:00 to 9:30 p.m. However, school children are at school during the daytime (about 9:00 a.m. to 2:00 p.m. for the first-grade elementary students in South Korea), and the sun is the most powerful light source during the day. Classroom illuminance is more strongly affected by sunlight than a lighting system during the day. Consequently, classroom illuminance is affected by weather and time and changes constantly. We used a daylight factor rather than daytime classroom illuminance, which tends to be constant regardless of outdoor brightness. The daylight factor reflects daytime classroom illu- 
minance in a more practical way. Additionally, the schools enrolled in our study were built to the Korean Standard (KS A 3011) in which the lowest illumination at the desk plane is 300 lux. Thus, our study reflected practical daytime classroom illuminance.

The mechanism of the decrease in myopic shift due to increased indoor light level is not clear. In our study, the classrooms had windows, and a large portion of classroom illuminance originated from sunlight. Thus, increased classroom illuminance would share the mechanism of decreased axial elongation by outdoor activity. In addition, hyperopic retinal blur due to a high lag of accommodation during near-viewing activities has been proposed as a cause of juvenile-onset myopia progression in humans [17,19] A lower classroom illuminance level may disturb pupil constriction and cause a lag of accommodation, subsequently increasing peripheral retinal defocus. Additional studies on the mechanism of the protective effect of indoor illumination on refractive error should be performed.

Some limitations in this study should be mentioned. Cycloplegic refraction was not performed, and the follow-up period was short. Only two schools with highest and LD factor were included. The presence of parental myopia, the amount of outdoor activity and near-work were evaluated by survey, which may have produced subjective results. Other factors related with myopia, such as parent education level and family income, were not investigated in this study [3]. Further study assessing children's genetic and environmental factors objectively with a longer follow-up period will be necessary.

In conclusion, high classroom illuminance including sunlight from windows during the daytime reduced axial elongation of the eye in the children with shorter AL. The increase in classroom light level by permitting more sunlight to enter might be protective against the development of myopia in addition to increasing outdoor activity.

\section{Conflicts of Interest: None.}

Acknowledgements: The authors are grateful to Dr. JongMin Lim and Hyo-Joo Kong of the Korea Institute of Lighting Technology for technical assistance.

Funding: This study was funded by the Chungcheongnamdo Office of Education.

\section{References}

1. Resnikoff S, Pascolini D, Mariotti SP, Pokharel GP. Global magnitude of visual impairment caused by uncorrected refractive errors in 2004. Bull World Health Organ 2008;86: 63-70.

2. Leo SW, Young TL. An evidence-based update on myopia and interventions to retard its progression. J AAPOS 2011;15:181-9.

3. Morgan IG, Ohno-Matsui K, Saw SM. Myopia. Lancet 2012;379:1739-48.

4. Chang RT, Singh K. Myopia and glaucoma: diagnostic and therapeutic challenges. Curr Opin Ophthalmol 2013;24:96101.

5. Ohno-Matsui K, Lai TY, Lai CC, Cheung CM. Updates of pathologic myopia. Prog Retin Eye Res 2016;52:156-87.

6. Holden BA, Fricke TR, Wilson DA, et al. Global prevalence of myopia and high myopia and temporal trends from 2000 through 2050. Ophthalmology 2016;123:1036-42.

7. Wallman J, Winawer J. Homeostasis of eye growth and the question of myopia. Neuron 2004;43:447-68.

8. Chua WH, Balakrishnan V, Chan YH, et al. Atropine for the treatment of childhood myopia. Ophthalmology 2006; 113:2285-91.

9. Cho P, Cheung SW, Edwards M. The longitudinal orthokeratology research in children (LORIC) in Hong Kong: a pilot study on refractive changes and myopic control. Curr Eye Res 2005;30:71-80.

10. Jones LA, Sinnott LT, Mutti DO, et al. Parental history of myopia, sports and outdoor activities, and future myopia. Invest Ophthalmol Vis Sci 2007;48:3524-32.

11. Lee YY, Lo CT, Sheu SJ, Yin LT. Risk factors for and progression of myopia in young Taiwanese men. Ophthalmic Epidemiol 2015;22:66-73.

12. Saw SM, Chua WH, Hong CY, et al. Nearwork in early-onset myopia. Invest Ophthalmol Vis Sci 2002;43:332-9.

13. Wu MM, Edwards MH. The effect of having myopic parents: an analysis of myopia in three generations. Optom Vis Sci 1999;76:387-92.

14. Sherwin JC, Reacher MH, Keogh RH, et al. The association between time spent outdoors and myopia in children and adolescents: a systematic review and meta-analysis. Ophthalmology 2012;119:2141-51.

15. Rose KA, Morgan IG, Ip J, et al. Outdoor activity reduces the prevalence of myopia in children. Ophthalmology 2008;115:1279-85. 
16. Wu PC, Tsai CL, Wu HL, et al. Outdoor activity during class recess reduces myopia onset and progression in school children. Ophthalmology 2013;120:1080-5.

17. Norton TT, Siegwart JT Jr. Light levels, refractive development, and myopia: a speculative review. Exp Eye Res 2013; 114:48-57.

18. Flitcroft DI. The complex interactions of retinal, optical and environmental factors in myopia aetiology. Prog Retin Eye Res 2012;31:622-60.

19. Ngo C, Saw SM, Dharani R, Flitcroft I. Does sunlight (bright lights) explain the protective effects of outdoor activity against myopia? Ophthalmic Physiol Opt 2013;33:36872.

20. Cohen Y, Belkin M, Yehezkel O, et al. Dependency between light intensity and refractive development under light-dark cycles. Exp Eye Res 2011;92:40-6.

21. Ashby R, Ohlendorf A, Schaeffel F. The effect of ambient illuminance on the development of deprivation myopia in chicks. Invest Ophthalmol Vis Sci 2009;50:5348-54.

22. Smith EL 3rd, Hung LF, Huang J. Protective effects of high ambient lighting on the development of form-deprivation myopia in rhesus monkeys. Invest Ophthalmol Vis Sci 2012;53:421-8.

23. Hua WJ, Jin JX, Wu XY, et al. Elevated light levels in schools have a protective effect on myopia. Ophthalmic Physiol Opt 2015;35:252-62.

24. McKnight CM, Sherwin JC, Yazar S, et al. Myopia in young adults is inversely related to an objective marker of ocular sun exposure: the Western Australian Raine cohort study. Am J Ophthalmol 2014;158:1079-85.

25. Mutti DO, Hayes JR, Mitchell GL, et al. Refractive error, axial length, and relative peripheral refractive error before and after the onset of myopia. Invest Ophthalmol Vis Sci 2007;48:2510-9. 